MODELING, IDENTIFICATION AND CONTROL, 1998, vOL. 19, NO. 4, 175-184

doi:10.4173/mic.1998.4.1

\title{
MPC in Systems with Continuous and Discrete Control Inputs
}

\author{
OLAV SLUPPHAUG $\dagger$, JOSTEIN VADA $\dagger$ and BJARNE A. FOSS $\dagger$
}

Keywords: Predictive control, hybrid control, logic design

In this paper we point out potential problems in systems where both logic and continuous control influence a continuous plant. We also propose model predictive control (MPC) based approaches for handling the problems. Simulations on a simple but nontrivial plant support the discussion.

\section{Introduction}

Industrial control systems are generally comprised of logic and continuous controllers. The logic performs functions like starting process equipment, controlling low-level (or regulatory) controllers, and controlling discrete control inputs (on/off valves, pumps etc.). The logic may for instance switch between different continuous controllers depending on the operating conditions of a process. Continuous controllers are used for regulatory control and for supervisory control. On the regulatory level PID-controllers constitute the typical continuous controllers. On the supervisory level continuous controllers compute the setpoints for the regulatory controllers using for instance an optimization algorithm. In our context it is important to note that the logic and continuous controllers in many cases are closely coupled, hence they form a hybrid dynamical system (HS). Typically, industrial design practice is based on a separate construction of the continuous and logic part of a control system. By this, the effect of the interaction between the continuous and logic part is difficult to foresee during the design-phase.

The hybrid nature of a system may also originate from the controlled system itself. In chemical process control a process may change its characteristics abruptly. One example of this is the change between laminar and turbulent flow conditions.

From the above observations we understand that, in general, a process system can be described as the HS in Fig. 1. Research in HS is conducted in both mathematical-, control-, and computer-science communities, see e.g. [1] and references therein. Research directed towards process control is reported in [2] and [3].

Model predictive control (MPC) has been an active research area for close to two decades. The research has been driven by numerous successful applications of the technology [4], and during the last years a sound theoretical foundation has been established [5], [6].

The use of MPC on HS is a possible way to integrate the design of the logic and continuous control and will be the topic of this paper. The hybrid nature is assumed to only be due to a mixture of continuous- and discrete-control inputs.

Received 2 September 1998.

†Norwegian University of Science and Technology, Department of Engineering Cybernetics. E-mail: Bjarne.A.Foss@itk.ntnu.no

This paper is a slight revision of a paper presented at the American Control Conference, Albuquerque, June 1997. Reprinted with permission from the American Automatic Control Council. 


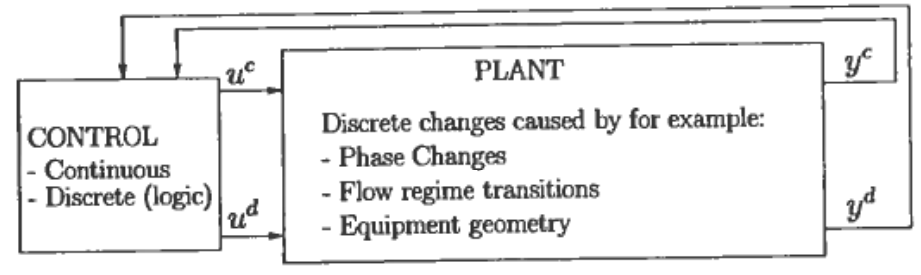

Figure 1. Process control. $u^{c}$ denotes the continuous control inputs, $u^{d}$ denotes the discrete control inputs, $y^{c}$ denotes the continuous outputs, and $y^{d}$ denotes the discrete outputs.

The scope of this paper is twofold. First, we show potential problems that may arise by designing the logic and continuous controller separately. Second, we investigate the use of MPC as a means for integrating the design of logic and continuous control. The presentation is based on the example described in the next section.

\section{Example}

The example system in Fig. 2 consists of two tanks; the buffer and the supply, and four control inputs; a two-stage pump, a continuous heater, and two on/off valves-one at the inlet of the buffer and one at the outlet of the supply. The function of the plant is to receive liquid from an upstream process, and to deliver this liquid at some reference temperature to a downstream process. The flowrate and temperature of the inflow to the buffer, and the flowrate demand from the downstream process are disturbances.

\subsection{Model}

If we assume constant liquid density, constant specific heat capacity of the liquid, and isolated tanks - mass and energy balances give the following set of differential equations describing the example system:

$$
\begin{aligned}
& \dot{x}_{1}=\frac{1}{A_{b}}\left(v_{1} u_{3}^{d}-\alpha u_{2}^{d}\right) \\
& \dot{x}_{2}=\frac{1}{A_{b} x_{1}}\left(-x_{2} v_{1} u_{3}^{d}+v_{1} v_{2} u_{3}^{d}\right) \\
& \dot{x}_{3}=\frac{1}{A_{s}}\left(\alpha u_{2}^{d}-v_{3} u_{4}^{d}\right) \\
& \dot{x}_{4}=\frac{1}{A_{s} x_{3}}\left(\left(x_{2}-x_{4}\right) \alpha u_{2}^{d}+\frac{u_{1}^{c}}{c_{l} \rho_{l}}\right),
\end{aligned}
$$

where $x=\left(x_{1}, x_{2}, x_{3}, x_{4}\right)$ is the state, $u=\left(u_{1}^{c}, u_{2}^{d}, u_{3}^{d}, u_{4}^{d}\right)$ is the control input, and $v=\left(v_{1}, v_{2}, v_{3}\right)$ is the disturbance input. Let $f: \mathbb{R}^{4} \times \mathbb{R}^{4} \times \mathbb{R}^{3} \rightarrow \mathbb{R}^{4}$ be defined by:

$$
\dot{x}=f(x, u, v) \text {. }
$$

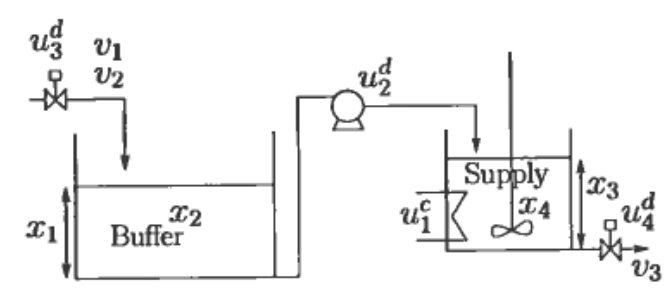

Figure 2. Two-tank system. 
Table 1. Model parameters

\begin{tabular}{lll}
\hline$A_{b}$ & $3.5 \mathrm{~m}^{2}$ & Buffer area \\
$A_{s}$ & $2 \mathrm{~m}^{2}$ & Supply area \\
$c_{l}$ & $4.2 \mathrm{~kJ} / \mathrm{kgK}$ & Specific liquid heat capacity \\
$\rho_{l}$ & $1000 \mathrm{~kg} / \mathrm{m}^{3}$ & Liquid density \\
$\alpha$ & $1 \mathrm{~m}^{3} / \mathrm{min}$ & Pump capacity factor \\
$\Delta$ & $0.25 \mathrm{~min}$ & Sample period \\
\hline
\end{tabular}

We use the following discrete-time system as the "real" system in the simulations:

$$
x(k+1)=x(k)+\Delta \cdot f(x(k), u(k), v(k)),
$$

i.e. the forward Euler numerical solution of (1) with step length $\Lambda$. For notational convenience we have let $x(k)=x(\Delta k)$ in (2). The model parameters are given in Table 1 , the legend for the states, controls, and disturbances is given in Table 2, and the nominal operating point is given in Table 3.

\subsection{Control objective}

The control task is to make the controlled system behave according to the following objectives:

- Prevent overflow/emptying of the buffer and the supply. Overflow is unwanted due to environmental/safety concerns, while emptying may cause malfunction of the heater $\left(u_{1}^{c}\right)$ and/or the pump $\left(u_{2}^{d}\right)$.

- The inlet- and outlet-valves should be open. Closing the inlet valve implies pollution as the inflow is rerouted to the environment. Closing the outlet valve leads to a downstream disturbance.

- Keep the supply temperature, $x_{4}$, at its nominal value, $x_{40}$.

Table 2. States, controls, and disturbances.

\begin{tabular}{ll}
\hline$x_{1}, x_{3}$ & Buffer and supply levels, respectively \\
$x_{2}, x_{4}$ & Buffer and supply temperatures, respectively \\
$u_{1}^{\mathrm{c}}$ & Heater (superscript $c$ means continuous) \\
$u_{2}^{d}$ & Pump (superscript $d$ means discrete) \\
$u_{3}^{d}, u_{4}^{d}$ & Inlet- and outlet-valves, respectivcly \\
$v_{1}$ & Inflow \\
$v_{2}$ & Temperature of inflow \\
$v_{3}$ & Outflow \\
\hline
\end{tabular}

Table 3. Nominal operating point.

\begin{tabular}{ll}
\hline$x_{10}$ & $7 \mathrm{~m}$ \\
$x_{20}$ & $18^{\circ} \mathrm{C}$ \\
$x_{30}$ & $1.5 \mathrm{~m}$ \\
$x_{40}$ & $22^{\circ} \mathrm{C}$ (setpoint) \\
$u_{10}$ & $280 \mathrm{~kW}$ \\
$u_{20}$ & $1 \mathrm{~m}^{3} / \mathrm{min}$ \\
$u_{30}$ & 1 (open) \\
$u_{40}$ & 1 (open) \\
$v_{10}$ & 1 (stage 1$)$ \\
$v_{20}$ & $18^{\circ} \mathrm{C}$ \\
$v_{30}$ & $1 \mathrm{~m}^{3} / \mathrm{min}$ \\
\hline
\end{tabular}




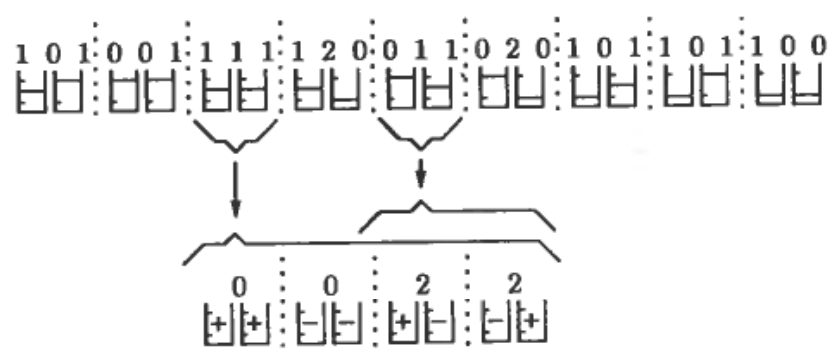

Figure 3. Generation of discrete control inputs. Upper part: The nine different possible combinations of high-, normal-, and low-levels in the buffer (left tank) and supply (right tank), separated by vertical dotted lines, are shown. The level limits (see Table 4) are indicated by two short horizontal lines in each tank. The three numbers above the tanks show, in each of the nine cases, the control input setting of the inlet valve $\left(u_{3}^{d}\right)$, the pump $\left(u_{2}^{d}\right)$, and the outlet valve $\left(u_{4}^{d}\right)$, respectively. Lower part: A " - " ("+ ") in the buffer means that $x_{2}(k)<x_{40}\left(x_{2}(k)>x_{40}\right)$. Correspondingly, a " +" (" - ") in the supply signifies that $x_{4}(k)>x_{40}+\frac{1}{2} \delta\left(x_{4}(k)<x_{40}-\frac{1}{2} \delta\right)$. If the level combination equals one of the two above the downward-facing braces in the figure, the pump will be used to speed up the temperature control if the temperature situation equals one of the combinations below the corresponding upward-facing braces. Then the pump stage is set to 0 or 2 (according to the present situation), while the valves keep their position from the upper part. See Table 4 for the value of $\delta$.

\subsection{Simulation scenario}

For time $k<0$, all variables are nominal. At time $k=0$, an unmodeled disturbance changes the supply temperature $\left(x_{4}\right)+2{ }^{\circ} \mathrm{C}$. All modeled disturbances $\left(v_{\mathrm{i}}\right)$ are assumed equal to their nominal values.

\section{Control strategies}

In this section we investigate the use of four different strategies for controlling the example system. All the strategies assume that all the states are measured. The strategies are compared with respect to the control objectives.

\subsection{Separate Design I}

As mentioned in the introduction, industrial design practice is often based on separate design of the continuous and logic part of the control system. In this and the next section, we consider two such designs. The logic and MPC data used in this design, Separate Design I, is described in the following.

\subsection{1. "Simple" logic}

The logic used in the example is shown in Fig. 3. Only the upper part of the figure is valid when "simple" logic is used.

Table 4. Logic control parameters.

\begin{tabular}{lll}
\hline$\delta$ & $0.3^{\circ} \mathrm{C}$ & Dead-band \\
$x_{1}^{l}$ & $1 \mathrm{~m}$ & Lower limit for buffer level \\
$x_{1}^{h}$ & $9 \mathrm{~m}$ & Upper limit for buffer level \\
$x_{3}^{l}$ & $1 \mathrm{~m}$ & Lower limit for supply level \\
$x_{3}^{h}$ & $9 \mathrm{~m}$ & Upper limit for supply level \\
\hline
\end{tabular}



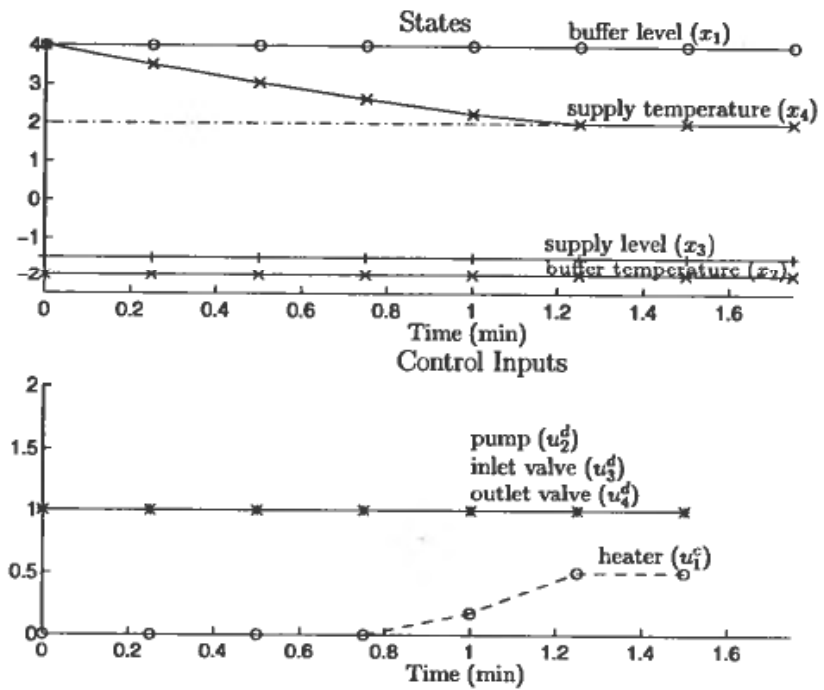

Figure 4. Separate Design I. The vertical axis in the upper part of the figure has the following interpretation: The zero point corresponds to $20^{\circ} \mathrm{C}$ and 3 meters for the temperature and levels respectively, and the scaling is $1^{\circ} \mathrm{C}$ and 1 meter respectively. The dash-dotted line is the setpoint $\left(x_{40}\right)$ for the supply temperature. Further, the vertical axis in the lower part of the figure has the following interpretation: The zero point corresponds to no heating, pump stopped, and closed valves. 1 corresponds to full heating $\left(2 u_{10}\right)$, pump running on stage 1 , and open valves. 2 corresponds to pump running on stage 2 .

\subsection{2. $M P C$ data}

Let $v_{0}=\left(v_{10}, v_{20}, v_{30}\right)$, and $u^{\mathrm{c}}=u_{1}^{c}$ and $u^{d}=\left(u_{2}^{d}, u_{3}^{d}, u_{4}^{d}\right)$ denote the continuousand discrete-part of the control input, respectively, i.e. $u=\left(u^{c}, u^{d}\right)$. Let $x(k+j \mid k)$ denote the predicted value of $x$ at time $k+j$ given information up to and including time $k$. For Separate Designs I \& II (for Separate Design II cf. Section 3.2) the MPC is based on the following optimization problem:

subject to

$$
\min _{\left(u^{c}(k \mid k), u^{c}(k+1 \mid k)\right)} \sum_{j=0}^{3}\left(x_{4}(k+1+j \mid k)-x_{40}\right)^{2},
$$

$$
\begin{aligned}
x(k+1+j \mid k) & =x(k+j \mid k) \\
& +\Delta \cdot f\left(x(k+j \mid k),\left(u^{c}(k+j \mid k), u^{d}(k-1)\right), v_{0}\right) \\
x(k \mid k) & =x(k) \\
0 & \leq u^{c}(k+j \mid k) \leq 2 u_{10} \text { for } j \in\{0,1\} \\
u^{c}(k+1 v k) & =u^{c}(k+2 \mid k)=u^{c}(k+3 \mid k) .
\end{aligned}
$$

In other words; the predictor is equal to the system (remember that the $v_{i} s$ stay at their nominal values), the prediction horizon is 4 , the move horizon is 2 , the (continuous) control input is kept at $u^{c}(k+1 \mid k)$ during the last two samples in the prediction horizon, and we assume full state measurement. Since we do not include knowledge about the logic design, we have chosen to use the last discrete control input, i.e. $u^{d}(k-1)$, as the discrete control input on the prediction horizon. This may be justified by a reasoning that the discrete control inputs normally change seldomly.

Let $\left(u^{c}(k \mid k)^{*}, u^{c}(k+1 \mid k)^{*}\right)$ denote the solution of the optimization problem, then, in these designs, the control input at time $k$ is given by:

$$
u(k)=\left(u^{c}(k \mid k)^{*}, \operatorname{logic}(x(k))\right) \text {, }
$$

where $\operatorname{logic}(\cdot)$ denotes the logic part of the control system. 

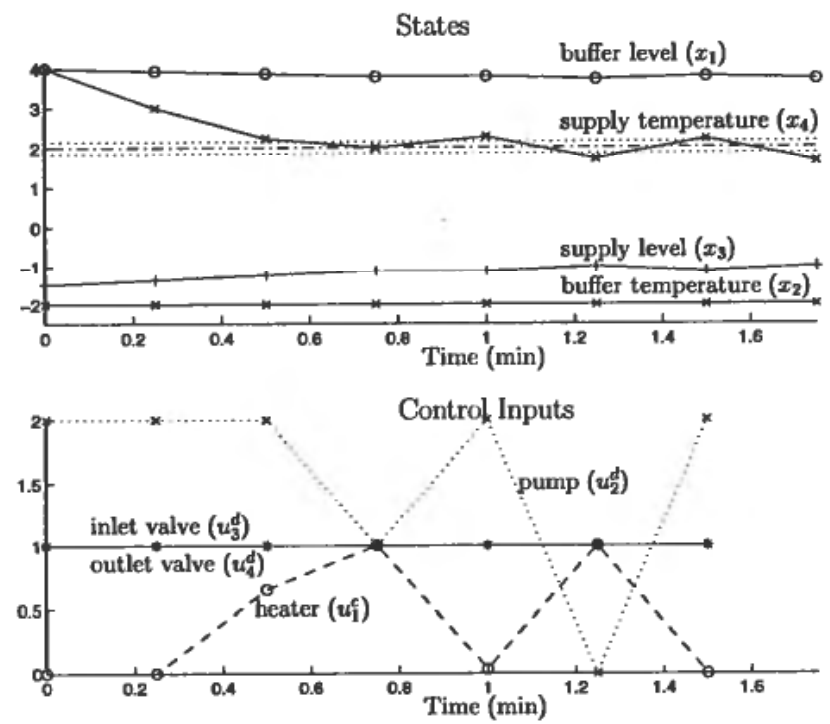

Figure 5. Separate Design II. For an explanation of the vertical axes, see Fig. 4.

\subsubsection{Simulation results}

Figure 4 shows the simulation results. The plot shows that the set point is reached after 1.25 minutes. In the following sections we propose strategies for improving the performance of the system, i.e. strategies for improving the disturbance attenuation.

In all the simulations the associated optimization problems are handled by the e04ucf routine (Num, 1995) for solving (locally) nonlinear programming problems.

\subsection{Separate Design II}

The performance of the system may increase if the pump is used in the temperature control in addition to the heater. For instance if the situation is that the levels in the two tanks are normal, and the temperature in the supply is above the setpoint $\left(x_{40}\right)$ and the buffer temperature is below $x_{40}$, the pump can be run on maximum speed. The logic and MPC data in this design, Separate Design II, is described in the following.

\subsection{1. "Advanced" logic}

The "advanced" logic is shown in the same figure as the "simple" logic, see Fig. 3. Now, the lower part of the figure is also valid.

\subsection{2. $M P C$ data}

The MPC is based on exactly the same optimization problem as for Separate Design $I$, cf. (3).

\subsubsection{Simulation results}

The simulation results are shown in Fig. 5. The plot shows that the system in this case will behave in an oscillatory manner. After 0.75 minutes $x_{4}$ enters the dead-band (from the upper side), and the pump is set to stage 1 by the logic $\left(u_{2}^{d}(3)=1\right)$. At the same time, the MPC computes the control input to the heater using $u_{2}^{d}(2)=2$ (the value at the previous sample) in the predictions. This leads to a control input to the heater, 

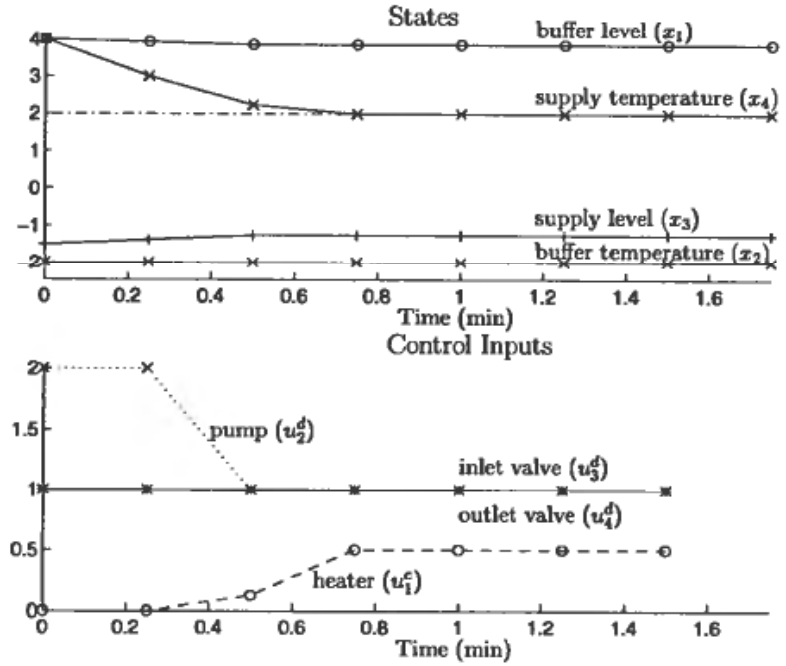

Figure 6. Full MPC. For an explanation of the vertical axes, see Fig. 4.

that is too large, which in turn causes $x_{4}$ to exit the dead-band upwards. As a result, the logic sets $u_{2}^{d}(4)=2$. At the same time, using $u_{2}^{d}(3)=1$ (the value at the previous sample) in the predictions, the MPC computes a control input value to the heater, that is too small, which in turn causes $x_{4}$ to cross the dead-band from the upper to the lower side. The behavior stays oscillatory.

The behavior of the system is strongly dependent upon the dead-band $(\delta)$. If $\delta$ is increased sufficiently, the oscillations can be damped or even removed completely. Note, however, that this new value for $\delta$ may not be sufficient to avoid oscillations at other operating points.

\subsection{Full $M P C$}

As noted above, tuning the parameter $\delta$ in Separate Design II is not easy. To avoid this tuning, we construct an MPC that computes all control inputs (continuous and discrete), Full MPC. The MPC data used is described next.

\subsubsection{MPC data}

Let $u_{0}^{d}=\left(u_{20}^{d}, u_{30}^{d}, u_{40}^{d}\right)$. In this strategy the MPC is based on the following optimization problem:

subject to

$$
\begin{aligned}
\min _{(u(k \mid k), u(k+1 \mid k))} & \sum_{j=0}^{3}\left(x_{4}(k+1+j \mid k)-x_{40}\right)^{2} \\
+ & \sum_{j=0}^{1}\left(u^{d}(k+j \mid k)-u_{0}^{d}\right)^{T} R\left(u^{d}(k+j \mid k)-u_{0}^{d}\right)
\end{aligned}
$$

$$
\begin{aligned}
x(k+1+j \mid k) & =x(k+j \mid k)+\Delta \cdot f\left(x(k+j \mid k), u(k+j \mid k), v_{0}\right) \\
x(k \mid k) & =x(k) \\
x_{1}^{\prime} & \leq x_{1}(k+j \mid k) \leq x_{1}^{h} \text { for } j \in\{1, \ldots, 4\} \\
x_{3}^{\prime} & \leq x_{3}(k+j \mid k) \leq x_{3}^{h} \text { for } j \in\{1, \ldots, 4\} \\
u(k+j \mid k) & \in\left[0,2 u_{10}\right] \times\{0,1,2\} \times\{0,1\}^{2} \text { for } j \in\{0,1\} \\
u(k+1+j \mid k) & =u(k+j \mid k) \text { for } j \in\{1,2\} \\
R & =\operatorname{diag}\{0.1,1,1\} .
\end{aligned}
$$



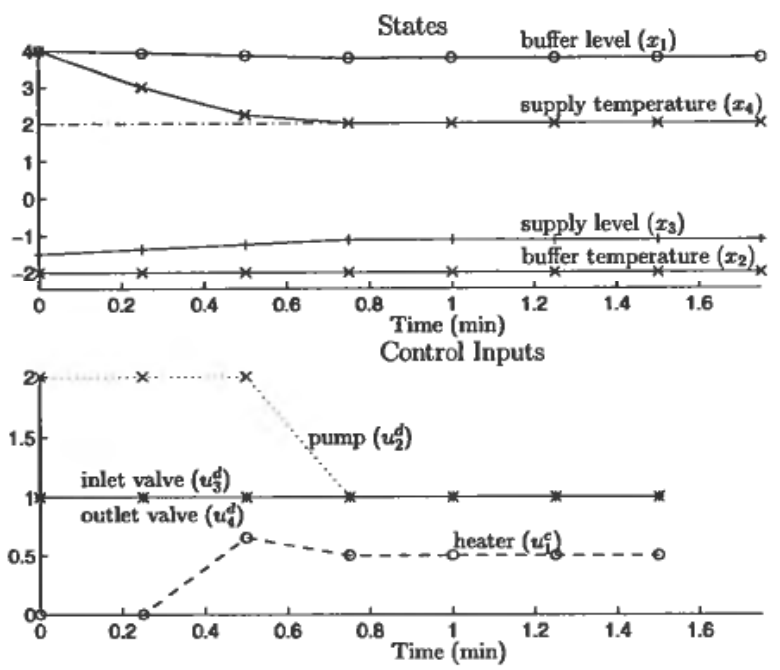

Figure 7. Integrated Design. For an explanation of the vertical axes, see Fig. 4.

\subsubsection{Simulation results}

Figure 6 shows the simulation results. The results show that the setpoint is reached after 0.75 minutes, i.e. a considerable improvement in performance compared to the two previous designs.

\subsection{Integrated Design}

Solving the full MPC problem in Section 3.3 is computationally intensive. (This is because the problem is a mixed-discrete nonlinear programming problem. We use a brute-force approach, and simply enumerate all possible discrete control input sequences and choose the one giving the lowest corresponding performance index when solving for the continuous control inputs.) It may be prohibitively intensive for on-line use. Also, the logic lies implicit in the Full MPC algorithm. This may not be desirable in an industrial environment where users are accustomed to an explicit logic description.

The above considerations indicate that in many cases it may be necessary to design the logic off-line. The logic may be designed by hand as in Section 3.1 or 3.2, or identified using Full MPC. To improve the performance of the systems presented in Sections 3.1 and 3.2 one can include the logic in the predictor used by the MPC. We denote this design Integrated Design. The logic presented in Section 3.2 is used, i.e. the "advanced" logic. Below, the MPC data used is presented.

\subsubsection{MPC data}

For this strategy, the optimization problem is equivalent to the one for Separate Designs I \& II, apart from the important difference that the logic is included in the predictor, cf. (4). We only state the new predictor:

$$
\begin{aligned}
x(k+1+j \mid k) & =x(k+j \mid k) \\
& +\Delta \cdot f\left(x(k+j \mid k),\left(u^{c}(k+j \mid k), \operatorname{logic}(x(k+j \mid k))\right), v_{0}\right) .
\end{aligned}
$$

\subsubsection{Simulation results}

Figure 7 shows the simulation results. The results show that the performance is improved compared to the results from Sections 3.1 and 3.2. The setpoint is reached after 0.75 minutes, which is the same as for full MPC (see Fig. 7). 


\section{Discussion}

The main purpose of this paper is to highlight possible problems which may arise in systems where there are both logic and continuous control influencing a continuous plant. As this is the case in most process control systems, these are important problems to be aware of.

In Separate Designs I \& II we chose to use the last discrete control input on the prediction horizon. An alternative could be to use nominal values for the discrete control inputs. We have also done this, and the results were qualitatively equivalent to the ones presented here.

As we see from the results of applying Separate Design I this may give a smooth behavior. This is also observed in many industrial systems. Looking more closely into Separate Design I, however, reveals that this design has a coupling from the logic control to the temperature control, but not vice versa. In Separate Design II we exploit the potential in the system by letting the temperature influence the logic control. This leads to a two-way coupling, with ultimately severe oscillations. These observations lead to the inference that today's industrial practice may be based on a conservative design philosophy in which the potential performance of systems may not be fully utilized. Moreover, it is no trivial task to design the logic so as to prevent two-way coupling. Hence, there is a need for looking into design methods where this type of two-way coupling is handled consistently. The last two strategies, Full MPC and Integrated Design, are suggestions for such design methods.

As noted above, one of the reasons for introducing the Integrated Design approach is a perceived need for explicit logic. The performance of the Integrated Design is, of course, dependent on the logic design, and in more complex cases than this example, it may be very hard to establish a good design simply because there exist no formal design methods. One approach to the logic design problem could be to generate data by simulating the Full MPC combined with an identification procedure for identifying a logic controller.

Another issue is how to solve the optimization problems arising. The Full MPC optimization problem is, in general, a mixed integer nonlinear programming (MINP) problem as mentioned above, while the optimization problem of the Integrated Design involves continuous variables and a discontinuous predictor. Neumaier (1996) seems to be a good starting point for further investigation of this very important issue.

\section{Conclusion}

We have shown potential problems that may arise by separately designing logic and continuous control, and proposed two MPC strategies as means for improved design. The potential problems/improvements are highlighted via simulations of a simple but nontrivial example. The two mentioned strategies, Full MPC and Integrated Design, seem to be good candidates for integrating the design of logic and continuous control.

REFERENCES

Alur, R., Henzinger, T.A. and SONTAG, E.D. (1996). Hybrid Systems III: verification and control, number 1066 in LNCS. Springer.

ENGELL S. and KOWALEWSKI, S. (1996). Discrete events and hybrid systems in process control. In: $C P C-V$. Tahoe City, CA, USA, January.

Lennartson, B., Tittus, M., Egardt, B. and Petterson, S. (1996). Hybrid systems in process control, Control Systems Magazine, October.

JOE QIN, S. and BADGWELL, T.A. (1996). An overview of industrial model predictive control technology. In: $C P C-V$. Tahoe City, CA, USA, January. 
Rawlings, J.B., Meadows, E.S. and MusKe, K.R. (1994). Nonlinear model predictive control: a tutorial and survey. In: Preprints IFAC Symposium ADCHEM, Kyoto, Japan.

LEE, J.H. (1996). Recent advances in model predictive control and other related areas. In: $C P C-V$. Tahoe City, CA, USA, January.

Num (1995). NAG Foundation Toolbox: For use with MATLAB.

NEUMAIER, A. (1996). Global and (Local) Optimization. http://solon.cma.univie.ac.at/ meum/ glopt.html 